\section{Mesodermal Wnt expression promotes liver specification}

Endodermal organs, such as liver and lung, arise at specific locations along the primitive gut tube. Many of the signalling pathways that specify endodermal organs have been determined, but no single gene that exclusively regulates liver specification has been identified. A recent study by Ober $e$ e al. (Nature, doi: 10.1038/nature04888; 2006) identified zebrafish mutants defective for early liver specification and suggests that mesodermal Wnt expression mediates this specification.

Ober et al. performed a genetic screen in a transgenic zebrafish line that expresses GFP in the developing endoderm. This identified a recessive mutant, prometheus ( $p r t$ ), in which the liver was absent or strongly reduced at 50 hours post fertilization (h.p.f.). By analysing liver markers, they observed that the defect in liver formation occurred very early in liver specification. The mutants had no other morphological phenotype, suggesting that prt is important for early liver development.

The group went on to determine where prt expression was required using mosaic analysis experiments. When wild-type cells were reintroduced into homozygous mutant embryos the prt mutant liver defect was rescued only if the wild-type cells were present in the lateral plate mesoderm (LPM) or the LPM and endoderm, but not in the endoderm alone. This indicates that the prt gene acts non-cell autonomously in liver development and is required in the mesoderm adjacent to where the liver, an endodermal organ, arises. This is the first in vivo demonstration that a signal from the mesoderm specifies liver fate and indicates that prt is required to mediate crosstalk between the LPM and the endoderm during liver formation.

Ober et al. then isolated the gene disrupted by the prt muation and identified a $w n t 2 b$ gene that they name $w n t 2 b b$. All three prt mutants that were identified had point mutations in this gene. Using

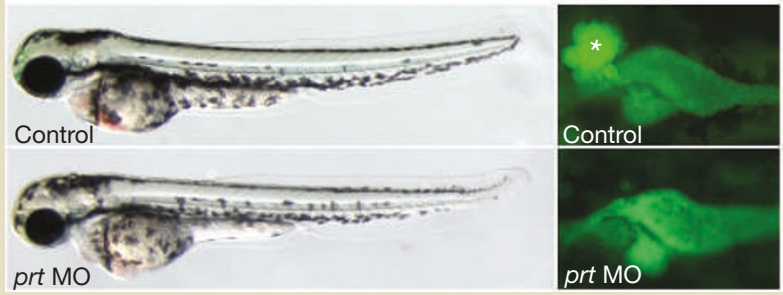

Knockdown of wnt2bb by prt $\mathrm{MO}$ led to a very strong reduction or complete absence of liver (asterisk in control), whereas the rest of the embryo seemed unaffected at 54 h.p.f.

morpholino oligonucleotide (MO) analysis they confirmed that wnt $2 b b$ is the gene affected in the prt mutants (see figure). The specific expression of $w n t 2 b b$ was investigated using cloche mutants (lacking endothelial cells) and casanova mutants (lacking all endodermal cells). Analysis of expression in these mutants determined that $w n t 2 b b$ is expressed in the pre-hepatic region of the LPM and that this expression does not depend on signals from the endoderm. Expression in this location is also consistent with the inductive properties of Wnt signalling.

The canonical Wnt signalling pathway, which acts through $\beta$-catenin, is known to specify endodermal organs, so the group investigated whether this pathway affects liver specifically. By inhibiting this pathway at various times, using a heat-shock inducible construct, they found that the critical time when $\beta$-catenin signalling was required is between 16 and 21 h.p.f., implicating this pathway in early liver development.

Taken together, these results indicate that the spatiotemporal expression of wnt $2 b b$ is consistent with its function in early liver specification. The work of Ober $e t$ al., and other recent studies, indicates that crosstalk between mesoderm and endoderm may contribute to the establishment of endodermal organs along the gut tube and that Wnt genes are key determinants for this induction.

SINÉAD HAYES 